\title{
El espacio público en el paradigma de la sustentabilidad social
}

\section{Public space in the paradigm of social sustainability}

\author{
Arturo Pérez-GonzÁlez*
}

\begin{abstract}
The construction of social relations has been traditionally generated through physicalresidential proximity. The neighborhood was the basis of neighbor and labor relationships as well as friendships. Therefore, public space has been a key supporting element for these social dynamics. However, the economic, technological, cultural and social changes of past decades have become new peripheral urban forms characterized by fragmentation and the absence of public space. For many authors, this represents a crisis of social sustainability. Nonetheless, this could be a paradigm shift, a new way to interact and socialize with space. From this perspective, the research conducted in the city of Guanajuato, has documented the spatial and sociofunctional model on which the social relationships of a sector of the population are currently built and held.
\end{abstract}

Keywords: Public space, social sustainability, paradigm.

\section{Resumen}

La construcción de relaciones sociales tradicionalmente se ha generado a partir de la proximidad físico-residencial. El barrio constituía la base de relaciones vecinales, laborales y de amistad, en este sentido, el espacio público representaba un soporte clave para las dinámicas sociales, pero los cambios económicos, tecnológicos y culturales de las últimas décadas han derivado en nuevas estructuras urbanas caracterizadas por la fragmentación espacial y carencia de espacio público; para algunos autores esto supone una crisis en la sustentabilidad social; sin embargo, podría significar sólo un cambio de paradigma, es decir, una nueva forma de interactuar con el espacio y socializar en él. Bajo esta perspectiva, la investigación realizada en la ciudad de Guanajuato, ha permitido documentar el modelo espacial y sociofuncional sobre el cual se construyen y sostienen actualmente las relaciones sociales de un sector de la población.

Palabras Clave: Espacio público, sustentabilidad social, paradigma.

* Alumno de posgrado Universidad del Bio-Bio, correo-e: arq.arturo.perez@gmail.com 


\section{Introducción}

Las ciudades son el hábitat dominante del ser humano desde finales del siglo pasado. Hoy, más de la mitad de la población mundial habita en ciudades, y su estudio es fundamental para la comprensión de los procesos de interacción social. Aunque los modelos de crecimiento urbano han sido intensamente explorados desde la década de 1920, es apenas en los últimos años que se han desarrollado estudios para las ciudades latinoamericanas (Borsdorf, 2003); sin embargo, suelen ser las grandes metrópolis las que captan la atención del investigador, sintetizando en algunos pocos modelos el comportamiento de la estructura urbana, por lo que en el caso de las ciudades medias los antecedentes son limitados (Álvarez-de la Torre, 2011), a pesar de que éstas, según datos del Instituto Nacional de Estadística y Geografía (INEGI) del 2001, tenían la mayor tasa de crecimiento y alojaban cerca de $46 \%$ de la población en México.

El crecimiento de las ciudades y un sistema económico caracterizado por la producción y consumo de grandes cantidades de recursos generó, a finales de la década de 1970, una preocupación por la capacidad de absorción del entorno; científicos, políticos y distintos sectores de la población estaban conscientes de la gravedad de los daños ambientales producidos al ecosistema, la idea de los recursos ilimitados desaparecía y daba lugar al temor de que los daños ocasionados pudieran ser irreversibles. Así, el crecimiento de las ciudades ha generado una preocupación por la vida urbana, llevando a cuestionar su viabilidad como hábitats sustentables.

Si bien los límites del crecimiento ya habían sido explorados por Thomas Malthus desde finales del siglo XVIII, quién había advertido sobre los peligros del aumento poblacional y la miseria, no es hasta 1972 que el Club de Roma ratificara a Malthus al publicar sus nada alentadores resultados en el informe denominado "los límites del crecimiento", en el cual se advertía que de seguir con la tendencia actual se estarían alcanzando los límites del planeta en los próximos cien años.

En este contexto, surge el concepto de "desarrollo sustentable", una idea conciliadora entre el desarrollo económico y la conservación del medio ambiente. El informe "Nuestro Futuro Común", redactado por la Comisión Mundial de las Naciones Unidas sobre Medio Ambiente y Desarrollo en 1987, definió el desarrollo sustentable como aquel que permite satisfacer las necesidades de las generaciones presentes sin comprometer las posibilidades de las generaciones del futuro para atender sus propias necesidades, así este concepto se incorporó en una gran cantidad de discursos, marcando un parteaguas en las políticas públicas de muchos países, especialmente en los más desarrollados. 
Aunque desde los años ochenta el concepto de la sustentabilidad reconocía una tridimensionalidad y un equilibrio entre la capacidad del medio ambiente, las unidades económicas y las aspiraciones humanas (Verstappen, 2009), la dimensión social estuvo todavía hasta finales de los noventa supeditada a la dimensión ecológica, abordando el concepto de manera simplista, y siendo presentada por muchos como un puente para frenar el deterioro medio ambiental (Colantonio, 2009), acusando los problemas de pobreza y crecimiento poblacional como los principales responsables de su degradación. Fue hasta finales del siglo xx que la comunidad internacional comenzó a plantearse como objetivo de la sustentabilidad social el crecimiento de las capacidades humanas, y el aumento de la calidad de vida, como un fin (Foladori, 2002).

Esta nueva visión del concepto dio lugar a un modelo teórico emergente que busca cambiar los tradicionales análisis e indicadores de la sustentabilidad social duros como la vivienda, el empleo o la satisfacción de otras necesidades básicas, por conceptos intangibles y menos cuantificables como la calidad de vida, el sentido de identidad o la felicidad; los modelos de evaluación emergentes son híbridos, dinámicos y multidimensionales, y se enfocan en los procesos, permitiendo evaluar los fenómenos sociales de manera interactiva; aunque de ninguna forma se deja de reconocer la importancia del medio físico y las necesidades básicas de la población como pilar de la sustentabilidad social (Colantonio, 2009).

La ciudad debería de concebirse como un espacio adecuado para la convivencia y la relación social. Las condiciones que conducen a la felicidad humana en toda su complejidad deben ser la preocupación central de las ciencias sociales (Colantonio, 2009). Se deben discutir las capacidades humanas y las relaciones sociales, no sólo por sus consecuencias técnicas como la pobreza o el desempleo, sino como la base de la participación y el empoderamiento, así como analizar la importancia del aumento de las cualidades y potencialidades humanas como un fin mismo (Foladori, 2002).

Richard Florida (2009), en su estudio sobre ciudades creativas señala que la clave de la felicidad son las relaciones afectivas, y que la capacidad de construir relaciones sociales genera bienestar y permite potencializar las capacidades humanas. Así, el concepto de la sustentabilidad social debería estar íntimamente ligado a la capacidad de construir relaciones sociales que permitan incrementar las capacidades humanas y alcanzar el desarrollo personal de los miembros de una comunidad.

Durante siglos, hemos construido nuestras relaciones afectivas a través de la proximidad, en este sentido, el barrio y el vecindario eran clave en este proceso, y los espacios públicos constituían el soporte principal de las relaciones sociales; sin embargo, desde finales del siglo pasado las ciudades han experimentado una transformación que ha dado paso a un 
modelo urbano fragmentado, donde los espacios públicos han pasado a ocupar un lugar residual en los nuevos desarrollos, dando lugar a lo que para algunos expertos representa una crisis de la sustentabilidad social y urbana. Entonces ¿cómo se construyen relaciones sociales bajo este modelo de ciudad donde el principal soporte de las relaciones sociales parece ausente?

Se plantea la hipótesis de que el paso de un modelo territorial compacto a uno disperso y reticulado ha fortalecido la integración de comunidades por interés común y capacidad de movilidad individual, en lugar de la proximidad física. La concepción y producción del desarrollo urbano periférico ha venido acompañada de nuevos patrones de sociabilización trans-espaciales, diversificando los soportes de las relaciones sociales, en especial de las clases medias.

No obstante, este cambio en la estructura de las relaciones sociales no supone la reducción de la sustentabilidad social; la ausencia de espacios públicos contiguos en los nuevos modelos urbanos, cómo se puede observar en los resultados de la presente investigación, no es necesariamente síntoma de un proceso de atomización social o de reducción del capital social, sino más bien un cambio interactuante a los nuevos modelos sociales, culturales y económicos que han dado paso a estructuras más complejas y soportes más diversos; los patrones de movilidad e interacción social encontrados en los habitantes de la ciudad de Guanajuato, sugieren un cambio de paradigma, es decir, una nueva forma de interactuar con la ciudad y socializar en ella. En el urbanismo moderno, el cambio hacia la construcción de una sociedad sustentable debe plantear nuevas formas de relacionarnos con la ciudad, acordes al modelo de relaciones sociales, culturales, económicas y productivas actuales. Las relaciones que sirven de soporte de la sociedad moderna hacen necesaria la búsqueda de nuevos paradigmas (López-Bernal, 2004).

\section{De la fragmentación urbana a la crisis del espacio público}

Durante los años veinte, el movimiento moderno concluyó que la mejor forma de organizar la ciudad era a través de la zonificación y la inserción de grandes vías que permitieran el traslado de sus habitantes de un lugar a otro. Las ideas de Le Corbusier permearon en los planes estratégicos de muchas ciudades, incluso aún se conservan en numerosos planes de ordenamiento territorial, pese a que fueran altamente criticadas desde los años sesenta. Durante las décadas de 1970 y 1980, el Estado asumió la responsabilidad de la planificación, poniendo en marcha diversos planes y ordenamientos que recogían muchas de las ideas del movimiento moderno, trayendo como consecuencia una sectorización de la ciudad. Pero 
las reformas económicas y políticas que se llevaron a cabo en Latinoamérica durante los años noventa fueron determinantes en la construcción de un nuevo modelo urbano sujeto a nuevas tendencias, las leyes del capitalismo.

El modelo económico y político de esta década, la prevalencia de modelos urbanos de los años veinte basados en la zonificación, el uso masivo del automóvil y el desarrollo de nuevas tecnologías de información y comunicación, así como la aparición de medios de transporte más rápidos, favoreció un modelo de ciudad disperso. Las actividades se distribuyeron en el territorio, consumiendo grandes cantidades de suelo y privilegiando la movilidad en el trasporte privado, con altos costos energéticos y medio ambientales. Las políticas públicas de la segunda mitad del siglo xx, en materia de vivienda, dieron paso a una morfología geosocial caracterizada por la segregación residencial y una dispersión de la infraestructura y los servicios (Malizia, 2011). Los procesos de urbanización privada y la tendencia al aislamiento de complejos habitacionales y comerciales se impusieron en la mayoría de las metrópolis latinoamericanas, y aún en ciudades medias (Janoschka, 2002).

La fragmentación urbana vino acompañada de la desaparición o escases del espacio público. Los fraccionamientos cerrados construidos en la periferia en estructuras insulares complicaron la labor del Estado para dotar de infraestructura y servicios. El espacio público se convirtió en un elemento residual de las nuevas urbanizaciones, perdiendo su carácter integrador (Borja, 1998). La fragmentación condujo a la pérdida de los espacios de referencia para la construcción social y para el sentimiento de pertenencia, trayendo en consecuencia una crisis del espacio público. Dejar la planificación en manos de la iniciativa privada generó un desarrollo periférico con "efectos sociales negativos", como la ruptura de las relaciones sociales, de amistad y cooperación (Hidalgo, 2004).

Los espacios públicos constituyen puntos de encuentro entre las personas, favoreciendo el intercambio, la mezcla sociocultural y la inclusión social, contribuyendo de esta forma a la construcción de ciudadanía en un contexto democrático donde se expresa la diversidad y se aprende la tolerancia (Borja y Muxi, 2000). Estos son importantes para la construcción de una ciudad cohesionada y sostenible, pues actúan como lugares de centralidad y creación de identidad (Borja y Castells, 1999), y son fundamentales en los procesos de construcción de relaciones sociales, integración sociocultural y como agentes catalizadores del sentido de pertenencia (Lindón, 2003). No obstante, el concepto del espacio público como elemento central para la construcción de las relaciones sociales y como lugar de formación ciudadana es relativamente nuevo, esta conceptualización, afirma Salcedo-Hansen (2002) es una creación de la clase 
burguesa utilizada para transformar el orden social anterior e incrementar sus libertades con respecto al poder del Estado.

La discusión que se ha generado sobre la pérdida de espacios públicos constructores de ciudadanía en favor de espacios "seudopúblicos" privatizados, se ha fundamentado en el supuesto de que la ciudad alguna vez aceptó la diversidad y el intercambio social más de lo que lo hace ahora; sin embargo, este principio es cuestionable pues la nueva economía basada en el consumo ha permitido también la incorporación de muchas minorías y grupos (raciales y sexuales, principalmente) que eran excluidos hace algunos años (Salcedo-Hansen, 2002). Los cambios económicos, políticos y sociales de la última era han marcado el pulso de las transformaciones urbanas; las ciudades se han expandido de manera fragmentada con una evidente ausencia de espacio público, pero han aparecido también nuevas estructuras privadas o semipúblicas que han absorbido muchas de las funciones que concernían a estos lugares, los avances tecnológicos, particularmente en el área de la información, la comunicación y el transporte también han generado nuevas formas de interacción social y han incentivado nuevos hábitos dentro de la sociedad.

Actualmente, los análisis de los efectos sociales están centrados en las externalidades negativas que las soluciones urbanas representan, como falta de equipamiento, espacios públicos, áreas verdes, pero no sólo la distancia y acceso a los servicios es un factor de calidad de vida, lo es también la posibilidad de construir relaciones sociales. Es claro que la forma de socializar se ha diversificado y que la relación con la ciudad se ha transformado, dando paso a modelos de interacción nunca antes vistos. Pero muchos autores han insistido en que este modelo de crecimiento fragmentado, la segregación derivada del proceso de urbanización y la "crisis de los espacios públicos" refuerza las distancias sociales (Castells, 2008), provoca la ruptura de las relaciones de amistad y cooperación (Hidalgo, 2011), dualiza y excluye, reduciendo la solidaridad y la redistribución social (Borja y Muxi, 2000), contribuye a crear una agorafobia que limita las posibilidades de cohesión social y la creación de sentido de identidad y pertenencia (Borja, 1998; Carrión, 2004), e incluso promueve la desaparición de la vida urbana a través de la sustitución de los espacios públicos por no-lugares (Auge, 1998). Constituyendo un problema de sustentabilidad local y global (Borja y Castells, 1999).

\section{La construcción de las relaciones sociales en la era de la movilidad}

En el pasado la sociedad estaba estructurada localmente, y la construcción de las relaciones sociales se sostenía básicamente en las ciudades, por lo 
que hoy se sigue partiendo de que la territorialidad es un atributo fundamental en este proceso (Webber, 2004). Pero la sociedad, cada vez más compleja, se transforma a un ritmo mucho más acelerado de lo que lo hace el espacio edificado. La transformación de la ciudad históricamente ha estado vinculada al desarrollo de los medios de transporte y almacenamiento, y con la revolución industrial se dio un cambio radical en las estructuras urbanas. La aparición del ferrocarril dio acceso en el siglo XIX a la movilidad en masa, el automóvil vino a permitir la concentración humana y la eficiencia del movimiento de individuos y mercancías durante el siglo xx. El transporte descentralizó la ciudad y dilató el territorio, aboliendo limitaciones espaciales, mientras que la luz eléctrica y el teléfono redujeron la necesidad de una ubicación central (Fishman, 2004), las telecomunicaciones transformaron la estructura del comportamiento social cambiando la experiencia del tiempo y el espacio (Choay, 2009). La aparición de nuevos medios de almacenamiento amplió los límites espaciales y temporales de los individuos, debilitando las comunidades locales (Ascher, 2004).

Estos cambios han incentivando la creación de comunidades espacialmente dispersas, donde la territorialidad ya no es atributo necesario para las relaciones sociales, y donde las clases medias han accedido a privilegios que antes eran exclusivos de clases altas (Webber, 2004). La pertenencia a las comunidades no se funda ya sobre la proximidad ni sobre la aglomeración de las ciudades, sino sobre el interés común (Choay, 2009). Las comunidades de intereses han venido a dejar casi obsoleto el concepto de ciudad, anacrónico en un sentido convencional, y hoy se habla de regiones urbanas más que de ciudades. La dilatación del territorio "resta importancia a la proximidad en la vida cotidiana: el barrio ha dejado de ser lugar de integración de las relaciones de amistad, familiares, profesionales y cívicas; los vecinos son cada vez menos los amigos, parientes y colegas" (Ascher, 2004: 28).

Las personas tienen hoy mayores libertades para escoger dónde vivir, o con quién relacionarse, lo que fortalece las agrupaciones por intereses comunes. Los vínculos se han diversificado y se crean con mayor facilidad, aunque son también más débiles y menos estables (Ascher, 2004). Richard Florida (2009) define dos tipos de relaciones sociales: los vínculos afectivos, que son los que mantenemos con nuestros familiares y amigos, fuertes y persistentes; y los enlaces sociales, vínculos débiles que conformamos a través de las redes sociales, son menos intensos pero más extendidos, aunque lo importante de ellos es que nos aportan información nueva que incentiva el crecimiento personal.

López-Bernal (2004) hace referencia a una pérdida de la relación entre la cultura y el territorio geográfico, como una característica de las 
culturas urbanas posmodernas. En la sociedad actual, no estamos atados a la identidad de un lugar, y en general, solemos cambiar muy a menudo de residencia, de acuerdo al momento de nuestra vida. La ciudad se desterritorializa en sus representaciones, las cuáles coexisten por encima de la ciudad construida de diversas formas: alegóricas, simbólicas, imaginarias; hoy existe flotando sobre el territorio otra ciudad, una ciberciudad desespacializada que releva en funciones a la ciudad física (Sarlo, 2010).

Las nuevas Tecnologías de la Información y la Comunicación (TIC) han permitido revalorar las experiencias sensitivas y generar más posibilidades de movilidad, pero también han propiciado un mayor interés del individuo por reunirse con otras personas. Las relaciones cara a cara no se han desvanecido ni deteriorado, sólo diversificado y extendido. La movilidad real y virtual no está causando la extinción de la ciudad o de los lugares, sino que está generando nuevas formas de ciudad y de lugar. Los individuos aspiran hoy a tener un mayor control sobre su privacidad y su entorno, y para eso necesitan moverse en el tiempo y en el espacio. En una sociedad moderna que separa y reúne individuos y no grupos, que es diversa y heterogénea, las personas tienen una múltiple pertenencia social. Las comunidades se estructuran en redes interdependientes, donde las personas se relacionan de manera individual en múltiples entornos (Ascher, 2004).

Por ello, el reto del nuevo urbanismo es adaptarse a la movilidad. En la ciudad contemporánea, altamente urbanizada, extensa y discontinua, es fundamental garantizar la accesibilidad a través del derecho a la movilidad. Este derecho es fundamental para garantizar la inclusión social y el desarrollo personal en una sociedad que se diversifica crecientemente, entre más móviles somos, más posibilidades tenemos, pero este derecho está precondicionado a otros derechos previos, las desigualdades culturales y económicas restringen las posibilidades de algunos sectores de la población. Hay que considerar que la movilidad tiene costos económicos, sociales y ambientales altos, pero también ofrece mayores posibilidades de realización y crecimiento personal al brindar al individuo experiencias nuevas que le permiten explorar sus potencialidades.

Para tratar de corroborar la hipótesis de que las relaciones sociales han pasado de un modelo territorial a un modelo de movilidad e interés común, se llevó a cabo un estudio en la ciudad media de Guanajuato, donde el objetivo era analizar el modelo espacial y sociofuncional sobre el cual se construyen y sostienen actualmente las relaciones sociales de los habitantes de fraccionamientos urbanos de clase media ubicados en la 
periferia. Algunos de los resultados de esta investigación se presentan a continuación.

\section{La ciudad fragmentada}

Guanajuato, al igual que muchas ciudades latinoamericanas, pasó de una estructura compacta a una dispersa. Desde su fundación en el siglo XVI hasta mediados del siglo xx, el crecimiento de la ciudad se dio de forma centralizada y compacta. Posteriormente, durante la década de 1960 y hasta la década de 1980 se dio un crecimiento policéntrico acelerado, caracterizado por la intensa planificación del Estado, la migración del campo a la ciudad y el cambio de vocación económica, que dará lugar a una peri-urbanización del centro. A partir de la década de 1990, el nuevo modelo de desarrollo se rige por los cambios en la estructura económica del país y las nuevas políticas de crecimiento urbano que generaron un desarrollo fragmentado, donde escasean los espacios públicos y predominan los fraccionamientos urbanos como estructuras insulares.

La ciudad de Guanajuato ha tenido en las últimas dos décadas un crecimiento acelerado hacia la zona sur y suroeste del territorio. Este crecimiento se vio detonado por la aparición de fraccionamientos urbanos entre 1990 y 2010, los cuales, en términos generales, han aparecido de manera dispersa sobre el territorio, principalmente en las localidades de Marfil y Yerbabuena, con carencia de equipamiento urbano y espacios públicos. Esta parte del territorio presenta características de irregularidad, el cambio de uso de suelo para la proyección de fraccionamientos habitacionales creó islas urbanas en entornos rurales, dio lugar a la aparición de asentamientos irregulares y generó conflictos viales, además de falta de continuidad urbana e infraestructura de servicios, de acuerdo al Plan de Ordenamiento Territorial de 2011.

Los desarrollos de vivienda que proyectó la iniciativa privada en la periferia atrajeron a mucha gente joven del centro de la ciudad, especialmente de la clase media. Las restricciones urbanas en la cabecera y la actividad económica orientada a los servicios educativos, de gobierno y turismo, hicieron más rentable cambiar los usos de suelo habitacional por comercial, que junto con la falta de oferta habitacional, provocaron una migración de las generaciones más jóvenes.

En 1990, la población del municipio era de 119 mil habitantes, mientras que la ciudad abarcaba 873 hectáreas (1384 si se contemplan las localidades de Marfil y Yerbabuena), para el 2000, Guanajuato alcanza los 153,364 habitantes, de los cuáles, 53\% vivía en la cabecera municipal, y para el 2010, el municipio llegó a 171,709 habitantes, con 42\% viviendo en la cabecera, y otro $22 \%$ en las localidades periféricas de Marfil y Yerba- 
buena, según resultados del INEGI (2011), constituyendo entre las tres, la zona urbana más poblada del municipio. El territorio de la ciudad ocupaba en ese momento 2,799 hectáreas. Desde la década de 1990, el crecimiento del área urbana ha sido realmente acelerado, duplicando su extensión, tal como se observa en la figura 1, pero con una densidad de población baja y una estructura fragmentada que ha llevado a cuestionar su sustentabilidad.

\section{Movilidad: nuevo modelo de interacción socioespacial de las clases medias}

La movilidad urbana ha dado paso a nuevas formas de interactuar con la ciudad y con otras personas. Si bien las condiciones de la estructura y el desarrollo urbano han consentido la tendencia hacia la fragmentación y la segregación, estos procesos han venido acompañados de nuevas prácticas sociales y aparatos de soporte. La diversificación de la actividad económica y la especialización del trabajo y el estudio han llevado a crear comunidades cada vez más diversas y extendidas.

Las nuevas estructuras de consumo y los actuales sistemas de movilidad han permitido integrar territorios urbanamente inconexos y aglutinar comunidades espacialmente dispersas. Los espacios públicos que funcionalmente ejercían de elementos de conexión urbana se ven diluidos en la magnitud espacial de los nuevos entornos urbanos.

El trabajo y el ocio son actividades que impulsan el desplazamiento humano, se ha formado en la ciudad de Guanajuato un sistema urbano extendido y zonificado, donde las personas que habitan la periferia de la ciudad suelen desplazarse para cubrir sus necesidades laborales, comerciales o de ocio: entre semana se da una movilidad más intensa, los fines de semana más dispersa. El trabajo y el estudio es el principal impulsor de la movilidad en días hábiles, en tanto la recreación familiar es la principal impulsora los fines de semana.

Mientras que la periferia se configura como una ciudad dormitorio, el centro es un espacio orientado al ocio y al consumo, los bares, cafés, restaurantes y centros comerciales son los principales puntos de atracción para la población. La Zona Metropolitana de León-Silao constituye no sólo un foco de movilidad laboral, sino también de ocio y servicios. Las grandes tiendas departamentales y los centros comerciales, los negocios especializados y servicios específicos atraen a un gran número de personas. En el mapa 2 se puede observar la red de interacciones que los miembros de una familia de la periferia tienen con su contexto urbano. La necesidad de ir al trabajo, a la escuela, de compras o realizar actividades de ocio 


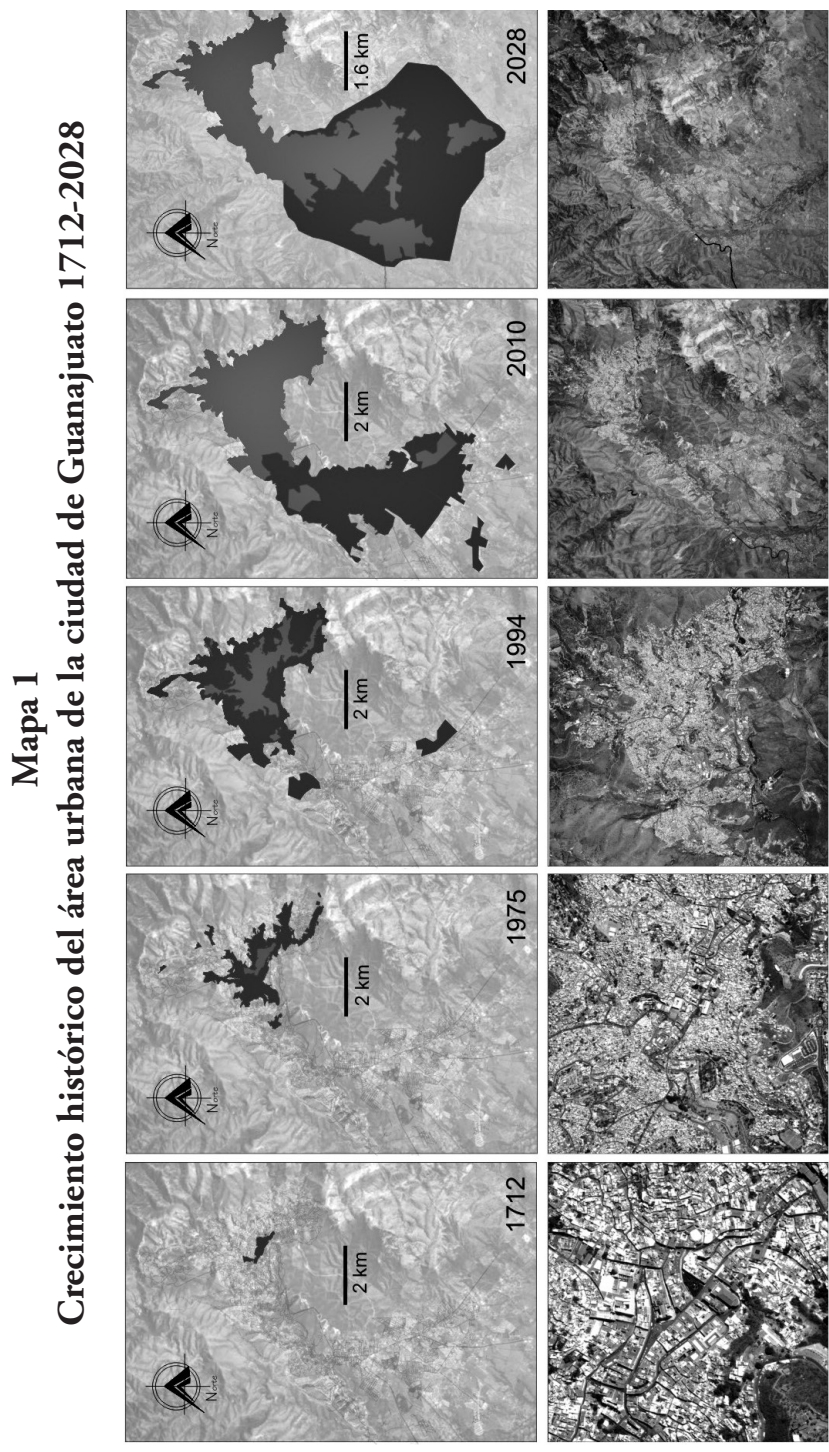

范

氙

:

尊 $\frac{0}{0}$

ㄴ.

ב.

过

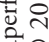

के ()

तु

気

के ज्ञ

$\approx$ O

ఏ సี

芯造

ชู 苂

ते $\dot{\Xi} \Xi$

를

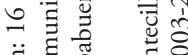

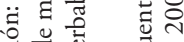

尊

워 $\overline{0}$

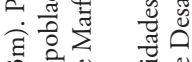

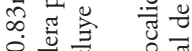

"I.

ส 0 ป

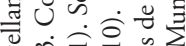

๘

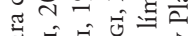

जै

远艺远芯

\& \&

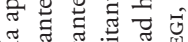

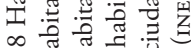

- 조 코

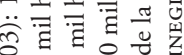

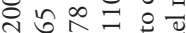

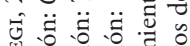

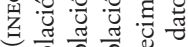

융 늉

毛实宁

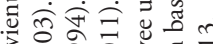

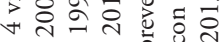

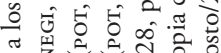

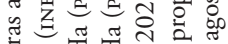

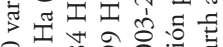
๖ ॠ

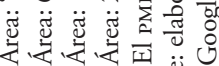

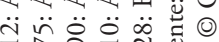

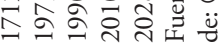


propicia recorrer la ciudad e incluso salir de ella. El marco de su interacción urbana se amplía tanto como sus necesidades y posibilidades de movilidad.

El uso de las TIC cobra alta importancia en los sistemas de redes de interacción social; el teléfono, sobre todo celular, permite a las personas interactuar a distancia y en cualquier momento con otras personas. Pero la deslocalización no se da en detrimento de los encuentros personales, sino que multiplica las interacciones sociales, los encuentros cara a cara son el segundo medio de comunicación para las personas sólo después del celular, tal como se observa en la gráfica 1 .

\section{Gráfica 1 \\ Medios de comunicación}

Medios de comunicación más utilizados por habitantes de fraccionamientos.

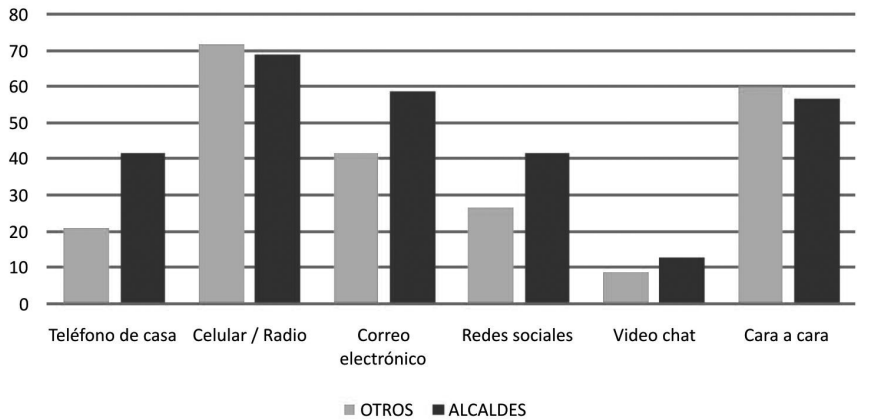

La gráfica muestra según sistema de puntuación la frecuencia con la que los encuestados usan cada uno de los distintos medios de comunicación.

Fuente: Elaboración propia con base en encuesta.

Los patrones de movilidad urbana permiten formarnos una idea de la amplitud territorial en la que se construyen las relaciones, pero también la intensidad y diversidad con que se generan. El fraccionamiento, el vecindario y el barrio se tornan cada vez menos significativos en la medida que las personas tienen acceso a la movilidad y pueden elegir con quien relacionarse. Los soportes de interacción social también se diversifican y se adaptan a las necesidades sociales; los espacios públicos, bares, cafés, centros comerciales, oficina o casa desempeñan roles distintos en la interacción social. Pero acaso esta expansión y diversificación de las relaciones sociales ¿se ha hecho en detrimento de la calidad de las relaciones afectivas?, ¿̇e han creado comunidades más débiles y apáticas?

\section{Relaciones sociales y afectivas: ¿̨proximidad o interés común?}

La movilidad urbana ha venido acompañada de un aparente ejercicio del individualismo, donde pareciera que las nuevas tecnologías han desalen- 


\section{Mapa 2 \\ Desplazamientos urbanos en habitantes de la periferia}

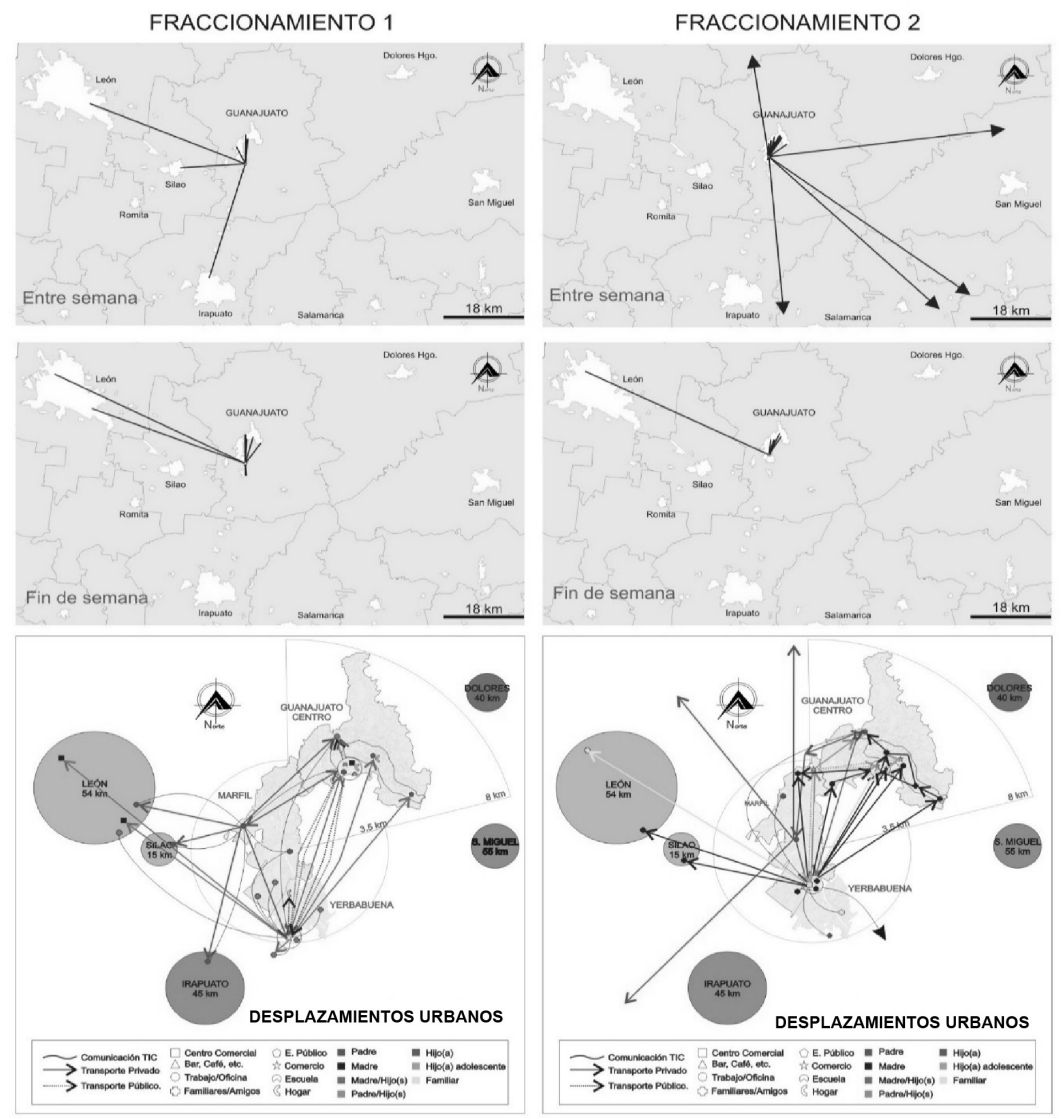

Fuente: elaboración propia a partir de información obtenida en entrevista a dos familias de la periferia.

tado los encuentros personales y vulnerado las relaciones afectivas. La desaparición del barrio como principal estructura comunitaria y la diversificación de los soportes de interacción social complican la observación de la construcción de relaciones afectivas. La dispersión concebida a través de los procesos de movilidad dificulta identificar las comunidades creadas a través de los intereses comunes, cuando el aspecto territorial parece ausente, especialmente cuando estas interacciones fluyen desde la intimidad de los espacios privados.

En la presente investigación, se encontró una tendencia de los habitantes de la periferia a construir y mantener relaciones sociales de mayor confianza fuera de su vecindario, los ámbitos familiares, de amistad y de trabajo son los de mayor confianza. La dispersión de las actividades laborales y la expansión urbana con el consiguiente esparcimiento de la red 
de relaciones afectivas, reduce los sistemas territoriales locales a interacciones más superficiales.

Los espacios públicos como la calle o la plaza soportan relaciones menos íntimas, mientras que los espacios semipúblicos o privados como cafeterías, restaurantes, lugares de trabajo y la casa habitación soportan aquellas relaciones de mayor intensidad. La zona metropolitana que actúa como centro de atracción y movilidad urbana no resulta tan significativa en los procesos de construcción de relaciones afectivas. La interacción con familiares y amigos se generan al margen de las prácticas de consumo y de ocio que las personas llevan a cabo con su familia nuclear. Los grupos comunitarios actúan en algunos casos como estructuras de ocio y recreación, y otras más como asociaciones con fines comunes, pero sin llegar a constituir comunidades de alta confianza. Las relaciones más fuertes y los procesos identitarios se estructuran a partir del interés familiar y laboral, incluso las relaciones de amistad se generan en ambientes laborales o estudiantiles antes que en el propio vecindario (gráfica 2).

\section{Gráfica 2 \\ Ambientes en que se generan nuevas relaciones de amistad}

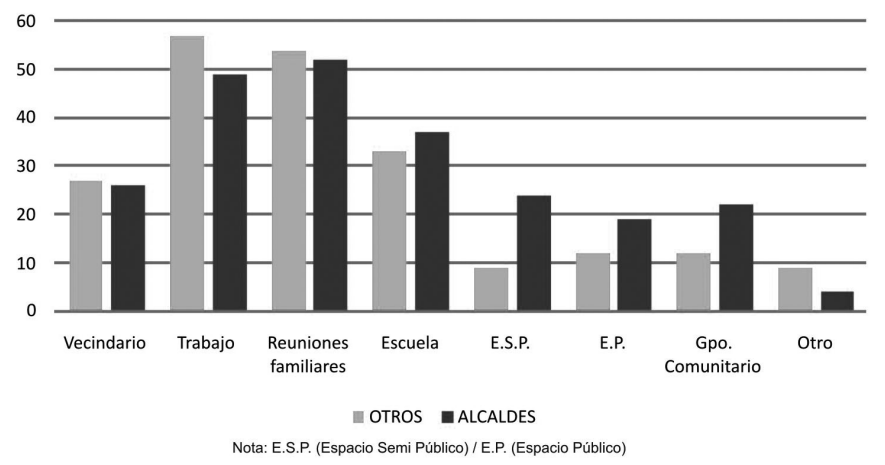

La gráfica muestra según sistema de puntuación, los ambientes en los que es más recurrente para los encuestados crear nuevas relaciones de amistad. Los ambientes familiares, de trabajo y educativos, son los más valorados.

Fuente: Elaboración propia con base en encuesta.

Esto se podría explicar debido a que las relaciones de amistad suelen estar subordinadas al interés común que presentan los miembros del grupo, el ambiente familiar constituye rasgos de identificación inherentes a la relación consanguínea que permiten ampliar la red de relaciones a través de las reuniones y encuentros familiares, mientras que los ambientes laborales y educativos son generadores de procesos de identificación individual con relación a la actividad profesional. El vecindario, por su parte, suele aportar pocos elementos para la identificación, incluso la presencia del espacio público no incentiva dicho sentimiento. En la grá- 
fica 3 se puede observar el nivel con el que los encuestados decían identificarse con un grupo social, tendencia que no se muestra alterada por las características físicas del vecindario.

\section{Gráfica 3 \\ Grupos con que las personas de los fraccionamientos se sienten más identificados}

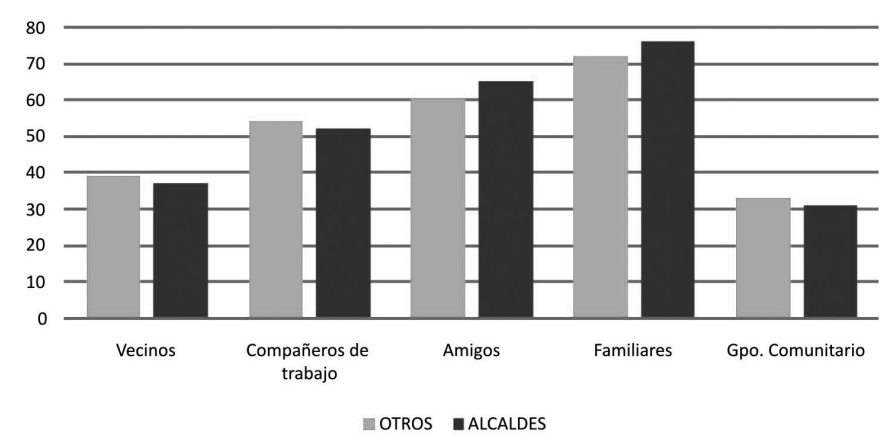

La gráfica muestra según sistema de puntuación, los grupos con los que las personas encuestadas se sentían más identificadas o con los que creían tener más intereses en común.

Fuente: Elaboración propia con base en encuesta.

Así, las relaciones de mayor confianza se generan en los círculos familiares y de amistad, y son soportadas en espacios privados o semipúblicos, estas relaciones son también espacialmente dispersas, pero se mantienen en gran medida con ayuda del transporte motorizado y las Tic, que permiten sostener relaciones afectivas de gran valor fuera incluso de la ciudad de residencia. El vecindario, en cambio, suele albergar relaciones de menor confianza, soportadas en ambientes públicos como la calle o la plaza, y no se observa que la presencia de espacios públicos consolidados o integrados al proyecto urbano cambie esta tendencia.

El mapa 3 resume de manera esquemática las redes de relaciones sociales de algunos de los habitantes de los Alcaldes, cruzando información referente a la relación social, la distancia, el soporte y la confianza en cada elemento del grupo. El análisis refleja que las relaciones de mayor confianza se generan a distancia, principalmente con familiares y amigos, mientras que las de menor confianza se dan dentro del vecindario. También se observa que los familiares, amigos y compañeros de trabajo comparten en menor medida el lugar de residencia.

Tampoco se encontró una correlación entre la calidad del espacio público con la formación de vínculos afectivos o relaciones sociales fuertes (gráfica 3); sin embargo, sí se observó que los niveles de confianza se correspondían con los niveles de identificación o afinidad que las personas decían sentir hacia los miembros de dicho grupo (gráfica, 4). En este 


\section{Mapa 3}

\section{Red de relaciones sociales en fraccionamiento los Alcaldes}
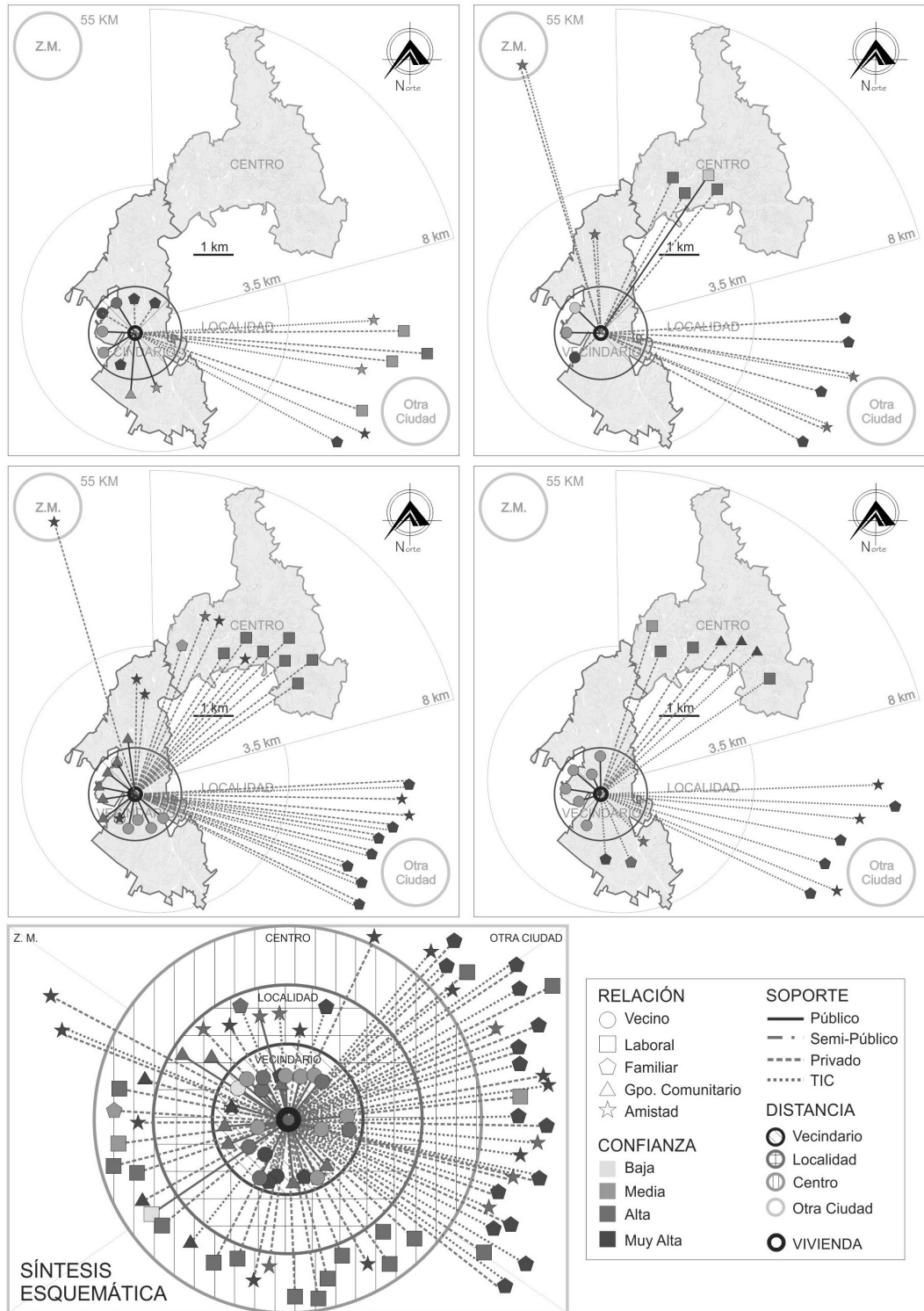

Gráfica de las relaciones sociales que sostienen habitantes de los Alcaldes obtenidos por entrevista. (Los puntos en el mapa hacen referencia a zonas espaciales, y no representan lugares concretos, en el último gráfico estas zonas se representan por circulos concéntricos de colores donde se sintetiza de manera esquemática los patrones de interacción.)

Fuente: Elaboración propia con base en encuesta. 
contexto, se comprobó que las relaciones de mayor confianza se generaban primero en el ámbito familiar, después en las relaciones de amistad, laborales, vecinales y finalmente en los grupos comunitarios; en el mismo orden que decían identificarse con los miembros del grupo.

\section{Gráfica 4}

\section{Calidad del espacio público $v s$. confianza en los vecinos}
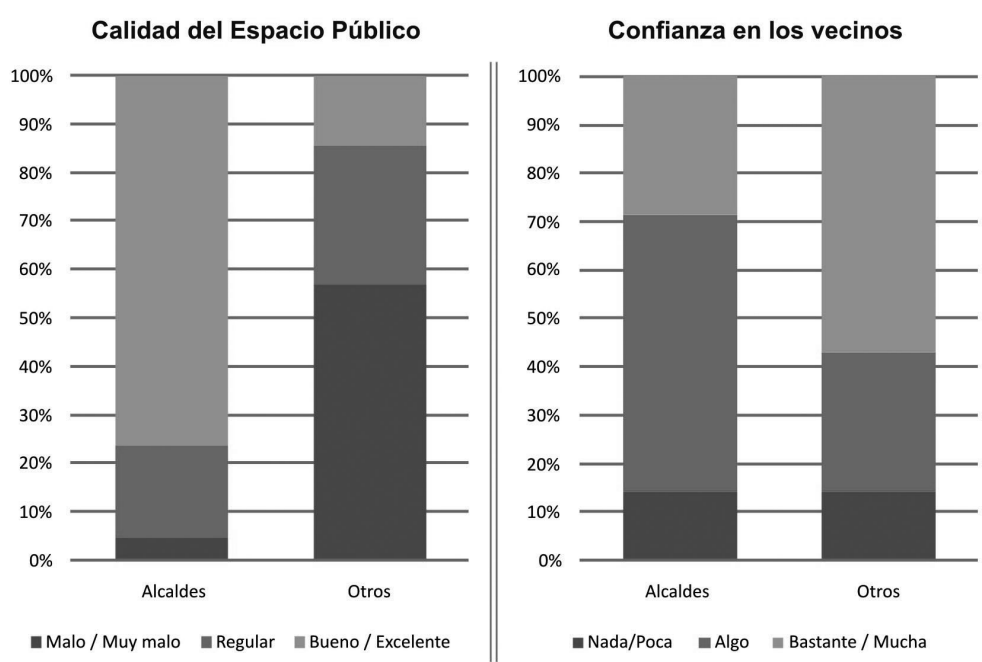

La gráfica muestra porcentualmente la percepción de la calidad de los espacios públicos de las personas encuestadas vs la confianza que decían tener en sus vecinos.

Estos datos nos permiten observar tres aspectos importantes, primero, que las estructuras barriales/vecinales pierden importancia cuando existe la posibilidad de construir relaciones sociales por interés propio, y cuando se tiene la capacidad de movilidad para elegir las relaciones afectivas. Una clase media con acceso a la movilidad preferirá aquellas relaciones surgidas por el interés común antes que las derivadas de la proximidad física.

Segundo, con el avance de la tecnología en materia de transporte y comunicación, el aspecto territorial (en los límites de las posibilidades de la movilidad) deja de ser determinante para la construcción de las relaciones sociales y afectivas, lo que permite construir y sostener vínculos afectivos a distancia, basados en el interés común. Estas nuevas comunidades son dispersas y reticuladas, y se soportan en estructuras muy diversas; las Tic, los medios de transporte motorizados y los espacios privados y semipúblicos se vuelven parte importante en los nuevos modelos de interacción social.

Tercero, el espacio público no constituye un elemento articulador de las relaciones sociales de alta confianza, y su calidad no fortalece los 
vínculos sociales en las comunidades vecinales cuando los miembros tienen acceso a la movilidad; sin embargo, su presencia si observa otras implicaciones.

\section{Gráfica 5}

\section{Confianza vs. identidad}
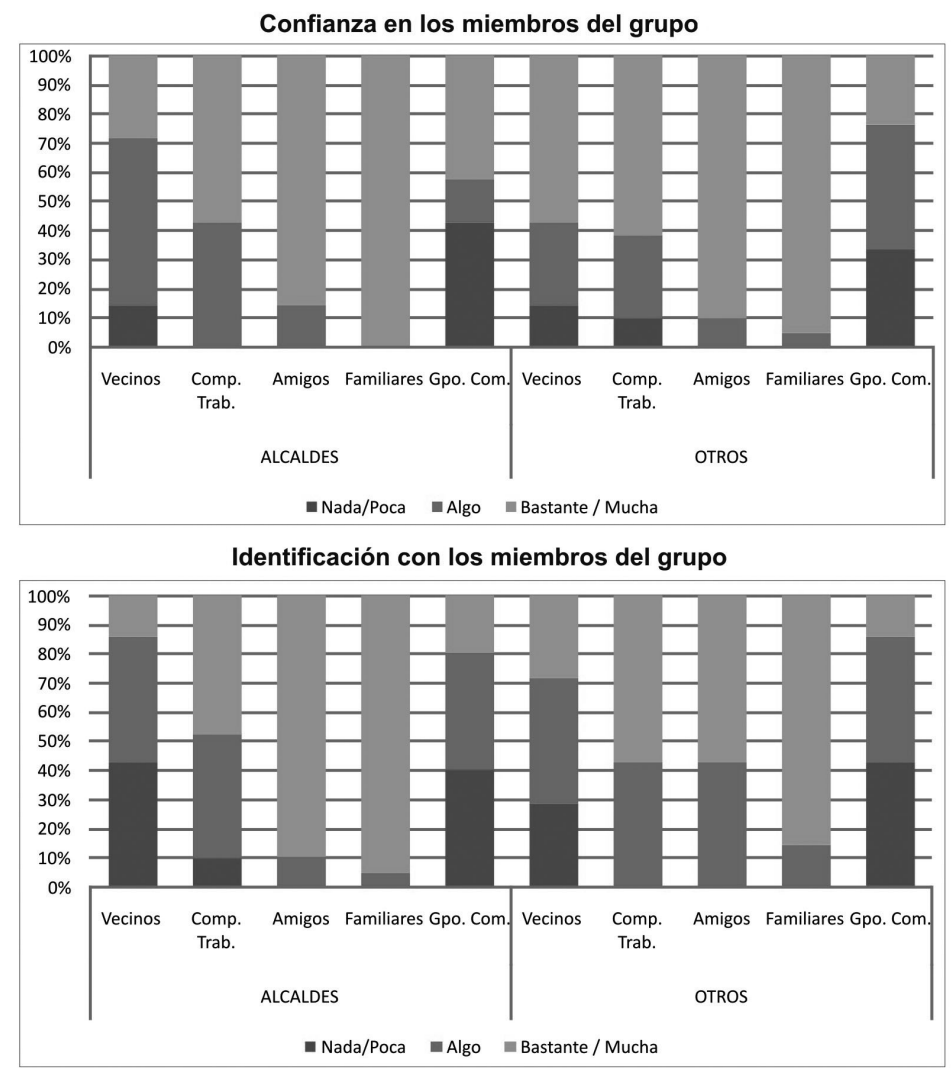

Las gráficas muestran porcentualmente, la confianza que los encuestados decían tener en los grupos a los que pertenecen, y comparativamente, qué tanto se sentian identificados con dichos grupos.

Fuente: Elaboración propia con base en encuesta.

\section{La mitificación del espacio público}

El espacio público solía constituirse como un soporte efectivo de las relaciones sociales porque estaban limitadas espacialmente y porque el modelo económico, derivado del liberalismo burgués, lo convirtió en expresión de libertades, derechos civiles y económicos; pero con los avances tecnológicos en materia de transporte y comunicación y ante la transformación del modelo económico, el espacio público ha venido delegando 
sus funciones en nuevas estructuras urbanas adaptadas a las actuales formas de vida y de consumo.

El espacio público ha adquirido una dimensión mitológica y romántica en el discurso de muchos urbanistas y actores políticos, al señalarlo como el gran constructor de las identidades sociales, pero constituye más un escenario donde se reflejan algunos procesos identitarios; las estructuras físico-espaciales son constructoras de identidades superficiales que nos permiten reconocer y reconocernos como parte de una forma urbana y de un territorio, pero estas identidades resultan menos efectivas en la construcción de vínculos fuertes; donde priman los ambientes familiares y de amistad formados desde la identificación por intereses comunes. Los procesos identitarios los construimos sobre bases menos tangibles, y desarrollamos identidades múltiples de pertenencia, que soportamos en medios menos visibles.

No obstante, no se puede argumentar como innecesaria la presencia del espacio público, pues éste alberga dinámicas sociales que favorecen otro tipo de vínculos, especialmente aquellos de convivencia, tolerancia y respeto; tampoco se puede seńalar que las interacciones sociales hayan perdido su soporte material, pues a pesar de los avances tecnológicos seguimos valorando los encuentros cara a cara y el contacto humano. El espacio público no ha dejado de ser importante, pero se debe de revalorar su función social, y considerar el impacto que tiene para los sectores menos móviles de la población; si bien, la población adulta de clase media y alta se vale de sus posibilidades de consumo y movilidad para entablar sus relaciones afectivas y sus interacciones sociales en ambientes semipúblicos o privados, grupos menos móviles como los niños tienen la necesidad de entornos próximos para interactuar, los jóvenes que tienen menor posibilidad de consumo también recurren a estos espacios como ambientes de recreación y ocio.

El estudio mostró que los principales ocupantes de estos espacios eran precisamente estos grupos de la población (gráfica 6). Esto invita a reflexionar sobre las características y los sectores de la población hacia los que debe estar orientado el espacio público de proximidad, en especial el de los fraccionamientos habitacionales.

En segundo lugar, se deben considerar los espacios públicos de los centros como caso de estudio aparte, pues a diferencia de los espacios de proximidad de los fraccionamientos urbanos, éstos constituyen estructuras de ocio, recreación y consumo en correlación con otros; además de que contienen una carga de simbolismo histórico que los hace diferenciarse. Los centros y, en especial, los centros históricos constituyen puntos de encuentro y áreas comerciales de gran valor, son vibrantes y atractivos; siguen vigentes y presentan la mayor frecuencia de uso y ocupación por 


\section{Gráfica 6 \\ Ocupación de los espacios públicos por grupos de edades}

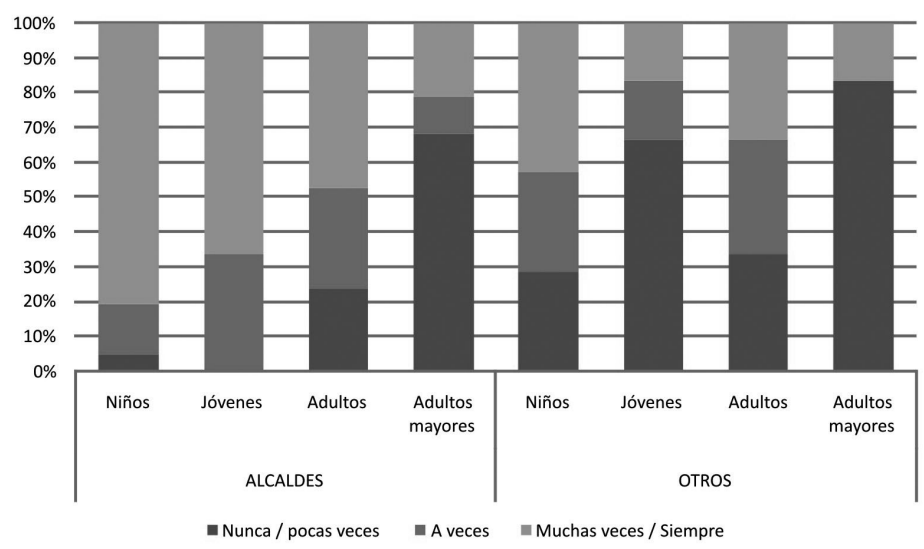

La gráfica muestra porcentualmente, la percepción que tienen los encuestados con respecto a los grupos de edades que utilizan con mayor frecuencia el espacio público de su fraccionamiento.

Fuente: Elaboración propia con base en encuesta.

los habitantes de la periferia, que los prefieren sobre otras estructuras de soporte emergentes como los centros comerciales, tal como se puede observar en la gráfica 7. La sociedad tiene la necesidad inherente de crear espacios de encuentro, éstos se han diversificado cada vez más en congruencia con los nuevos modelos culturales y estilos de vida.

\section{Gráfica 7}

\section{Frecuencia con que los habitantes de los fraccionamientos utilizan diversos espacios}

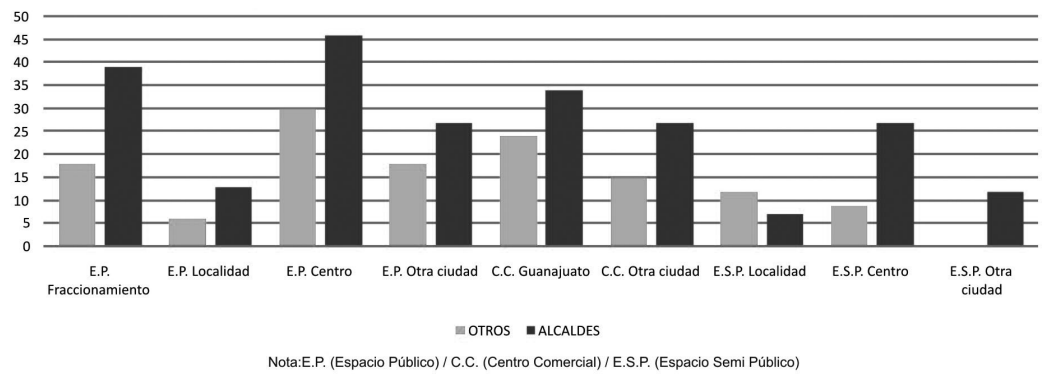

La gráfica muestra según sistema de puntuación, la frecuencia con la que los encuestados decian acudir a distintos espacios de la ciudad o de otra ciudad.

Fuente: Elaboración propia con base en encuesta. 


\section{Conclusiones}

El paradigma de la sustentabilidad social y del desarrollo urbano sustentable pasa necesariamente por identificar el soporte (o los soportes) de la interacción humana, cada vez más diverso y complejo, y esto nos lleva a cuestionarnos: ¿Se debe de seguir pensando la ciudad desde modelos territoriales, donde el espacio público contiguo asegure la capacidad de interacción social, o debemos dar paso a estructuras donde la movilidad genere las posibilidades de construcción de relaciones sociales desde la individualidad y los intereses comunes?

Las estrategias gubernamentales en materia de desarrollo social apuntan a lo primero, a reforzar las comunidades locales y buscar la mejora de la calidad de vida a través de la apropiación espacial, la participación, el empoderamiento y el rescate de los espacios públicos. Durante el sexenio anterior se invirtió una suma importante de recursos públicos a programas de rescate de espacios urbanos. Una estrategia dirigida a grupos sociales vulnerables con escasa posibilidad de movilidad; sin embargo, hay un reconocimiento implícito en algunas acciones gubernamentales que reconoce la importancia y la necesidad de la movilidad. La subvención del transporte público a algunos sectores de la población como niños, ancianos y estudiantes es una forma de incluir a estos grupos en el ejercicio del derecho a la ciudad. Algunos de los proyectos y esfuerzos por tener redes de datos abiertas en plazas públicas es un intento de inclusión social a través del acceso al ciberespacio.

Aunque los antecedentes y estudios similares a éste son limitados, los resultados obtenidos en esta investigación permiten aventurar que las clases medias han diversificado los soportes de su interacción social, demostrando que cuando existe la posibilidad de elegir, la importancia de los espacios de proximidad se reduce y se privilegian las relaciones generadas a partir del interés común. Por lo tanto, el espacio público toma nuevas dimensiones como aparato de soporte en las relaciones sociales, y su ausencia en el nuevo modelo de ciudad fragmentada, no imposibilita la construcción de las relaciones afectivas, las cuáles se generan a través de la movilidad.

Esto supone una nueva forma de mirar el urbanismo y concebir los espacios. El espacio público cobra nuevas dimensiones en el paradigma de la sustentabilidad social puesto que han aparecido estructuras que lo relevan en muchas de sus funciones, y esto amerita nuevas formas de entender ( $y$ atender) el diseño del espacio. Existe una urgencia por revalorar los espacios públicos y hacerlos funcionales a los cambios sociales y culturales de los nuevos modos de vida. La vida en la ciudad es hoy ostensiblemente más cara de lo que era hace algunos años, lo que presume 
implicaciones ambientales, económicas y sociales. La fragmentación urbana tiene costos energéticos altos y esto ha creado una clara tendencia en los discursos académicos actuales a resaltar los efectos sociales negativos de los actuales modelos de urbanización, pero sin reparar en los beneficios inherentes a estos cambios en los modos de vida y las posibilidades que ofrecen.

Pero hay que ser mesurados, porque estos procesos aún no incluyen a amplios sectores de la población, para quienes la proximidad física es el único soporte, ésta sigue siendo una base sólida para los que menor posibilidad tienen de moverse, incluyendo a niños y ancianos, no sólo clases marginadas. Las limitaciones de esta investigación están ceñidas a los resultados de estudiar una clase media con posibilidades de movilidad y bajo una estructura urbana determinada, por lo que hacen falta elementos de comparación y contraste. Plantear el tema de la sustentabilidad social, implica forzosamente centrarse en alcanzar estándares de felicidad y calidad de vida bajo formas urbanas inclusivas. Sin perder de vista que resolver los problemas de inequidad social se vuelve parte fundamental en la construcción de un hábitat sustentable, que ofrezca a todos los seres humanos la posibilidad de elegir con respecto a sus relaciones sociales, fortaleciendo los vínculos afectivos y de cooperación; favoreciendo el crecimiento personal y colectivo a través de la integración de comunidades con intereses comunes.

\section{Fuentes consultadas}

Álvarez-de la Torre, Guillermo (2011), "Estructura y temporalidad urbana de las ciudades intermedias en México", Frontera Norte, XXIII (46), El Colegio de la Frontera Norte, Tijuana, pp. 91-124.

Ascher, François (2004), Los nuevos principios del urbanismo, Alianza, Madrid.

Auge, Marc (1998), Los no lugares, espacios del anonimato: una antropología de la sobremodernidad, Gedisa, Barcelona.

Borja, Jordi (1998), "Ciudadanía y espacio público", Ambiente y Desarrollo, LXIV (3), Pontificia Universidad Javeriana, Bogotá, pp. 13-22.

Borja, Jordi y Manuel Castells (1999), Local y global. La gestión de las ciudades en la era de la información, Santillana, Madrid. 
Borja, Jordi y Zaida Muxi (2000), Espacio público, ciudad y ciudadanía, Electa, Barcelona.

Borsdorf, Axel (2003), "Cómo modelar el desarrollo y dinámica de la ciudad latinoamericana", EURE, XXIX (86), Pontificia Universidad Católica de Chile, Santiago, pp. 37-49.

Carrión, Fernando (2004), "Espacio público: punto de partida para la alteridad”, en F. Velázques, Ciudad e inclusión: por el derecho a la ciudad, Fundación Foro Nacional por Colombia, Bogotá, pp. 55-79.

Castells, Manuel (2008), La cuestión urbana, Siglo XXI, México.

Choay, Françoise (2009), "El reino de lo urbano y la muerte de la ciudad", Andamios. Revista de Investigación Social, VI (12), Universidad Autónoma de la Ciudad de México, México, pp. 157-187.

Colantonio, Andrea (2009), "Social sustainability: a review and critique of traditional versus emerging themes and assessment methods", en Horner, M. et al., (eds.) Sue-Mot Conference 2009: Second International Conference on Whole Life Urban Sustainability and Its Assessment: Conference Proce, Loughborough University, Loughborough, pp. 865-885.

Fishman, Robert (2004), "Más allá del suburbio: el nacimiento del tecnoburbio", en A. Ramos, Lo urbano en 20 autores contemporáneos, Universidad Politécnica de Cataluña, Barcelona, pp. 35-47.

Florida, Richard (2009), Las ciudades creativas. Por qué donde vives puede ser la decisión más importante de tu vida, Paidós, Barcelona.

Foladori, Guillermo (2002), “Avances y límites de la sustentabilidad social”, Economía, Sociedad y Territorio, III (12), El Colegio Mexiquense A. C., Zinacantepec, pp. 621-637.

H. Ayuntamiento de Guanajuato (2011), "Plan de Ordenamiento Territorial de Guanjuato Capital 2011”, Instituto Municipal de Planeación (Implan) Guanajuato, Periódico Oficial del Gobierno del Estado de Guanajuato, 21 de septiembre de 2012, Guanajuato. 
Hidalgo, Rodrigo (2004), "De los pequeños condominios a la ciudad vallada: las urbanizaciones cerradas y la nueva geografía social en Santiago de Chile (1990-2000)", EURE, XXX (91), Pontificia Universidad Católica de Chile, Santiago, pp. 29-52.

Hidalgo, Rodrigo (2011), "La urbanización en las áreas periféricas de Santiago y Valparaíso: El papel de las relaciones de poder en el dibujo de la geografía socioresidencial”, EURE, XXXVII (111), Pontificia Universidad Católica de Chile, Santiago, pp. 79-105.

INEGI (Instituto Nacional de Estadística y Geografía) (2011), XXIII Censo de población y vivienda 2010, INEgi, México, <www. inegi.org.mx>, julio de 2013.

INEGI (Instituto Nacional de Estadística y Geografía) (2003), Ciudades capitales, INEGI, México, <www.inegi.org.mx/geo/contenidos/ urbana/ciud_cap.aspx>, julio de 2013.

INEGI (Instituto Nacional de Estadística y Geografía) (2001), XXII Censo de población y vivienda 2000, INEGI, México, <www.inegi.org. $m x>$, julio de 2013 .

INEGI (Instituto Nacional de Estadística y Geografía) (1991), XI Censo de población y vivienda 1990, INEGI, México, <www.inegi.org.mx>, julio de 2013.

Janoschka, Michael (2002), "El nuevo modelo de la ciudad latinoamericana: fragmentación y privatización”, EURE, XXVIII (85), Universidad Pontificia Católica de Chile, Santiago, pp. 11-29.

Lindón, Alicia (2003), "Utopías, atopías y construcción del lugar”, Ciudades, núm 60, Universidad Autónoma Metropolitana, México, pp. 48-54.

López-Bernal, Oswaldo (2004), "La sustentabilidad urbana", Bitácora, 1 (8), Universidad Nacional de Colombia, Bogotá, pp. 8-14.

Malizia, Matilde (2011), "Enfoque teórico y conceptual para el estudio de las urbanizaciones cerradas", Andes, núm. 22, Universidad Nacional de Salta, Salta, pp. 293-318. 
Plan Municipal de Desarrollo 2003-2028 (2003), H. Ayuntamiento de Guanajuato, Guanajuato.

Salcedo-Hansen, Rodrigo (2002), "El espacio público en el debate actual: Una reflexión crítica sobre el urbanismo postmoderno", EURE, XXVIII (84), Universidad Pontificia Católica de Chile, Santiago, pp. 5-19.

Sarlo, Beatriz (2010), La ciudad vista, Siglo XXI, Buenos Aires.

Verstappen, Herman Theodoor (2009), "Geography, sustainability and the concept of glocalization”, Investigaciones Geográficas, núm. 70, Instituto de Geografía, México, pp. 106-113.

Webber, Melvin (2004), “La era postciudad”, en A. Ramos, Lo urbano en 20 autores contemporáneos, Universidad Politécnica de Cataluña, Barcelona, pp. 13-23.

Recibido: 3 de abril de 2014. Reenviado: 7 de octubre de 2014. Aceptado: 25 de noviembre de 2014.

Arturo Pérez-González. Mexicano. Licenciado en arquitectura por la Universidad de Guanajuato, México. Magíster en hábitat sustentable y eficiencia energética por la Universidad del Bio-Bio, Chile. Actualmente es arquitecto proyectista en el taller de arquitectura $G+G$ en la ciudad de Guanajuato. Ha colaborado en diversas publicaciones, tal es el caso de "El paisaje urbano y los reproductores de música en el Centro Histórico de la ciudad de Guanajuato", Revista electrónica Presencia, Universidad de Guanajuato, Guanajuato (2007). 